\title{
Prevalence of the Helicobacter pylori babA2 Gene in Children Mainly Depends on the PCR Primer Set Used
}

\author{
Anja Šterbenc, ${ }^{1}$ Maja M. Lunar, ${ }^{1}$ Matjaž Homan $\mathbb{D}^{0},{ }^{2}$ Boštjan Luzar, ${ }^{3}$ Nina Zidar, ${ }^{3}$ \\ and Mario Poljak ${ }^{1}$ \\ ${ }^{1}$ Institute of Microbiology and Immunology, Faculty of Medicine, University of Ljubljana, Zaloška 4, Ljubljana 1000, Slovenia \\ ${ }^{2}$ Department of Gastroenterology, Hepatology and Nutrition, University Children's Hospital, Faculty of Medicine, \\ University of Ljubljana, Vrazov Trg 2, Ljubljana 1000, Slovenia \\ ${ }^{3}$ Institute of Pathology, Faculty of Medicine, University of Ljubljana, Korytkova 2, Ljubljana 1000, Slovenia
}

Correspondence should be addressed to Mario Poljak; mario.poljak@mf.uni-lj.si

Received 11 June 2020; Revised 20 July 2020; Accepted 28 July 2020; Published 13 August 2020

Academic Editor: Peter J. Weina

Copyright (C) 2020 Anja Šterbenc et al. This is an open access article distributed under the Creative Commons Attribution License, which permits unrestricted use, distribution, and reproduction in any medium, provided the original work is properly cited.

\begin{abstract}
Various polymerase chain reaction- (PCR-) based methods with varying positivity rates were designed to detect the Helicobacter pylori babA2 gene. To compare different primer sets, babA2 prevalence was determined in $279 \mathrm{H}$. pylori-positive pediatric samples using the $832 \mathrm{bp}, 139 \mathrm{bp}$, and $271 \mathrm{bp}$ PCR primer sets, resulting in 34.0\%, 51.3\%, and 79.6\% prevalence of the babA2 gene, respectively. The babA2 status determined using the $832 \mathrm{bp}$ and $139 \mathrm{bp}$ PCR primer sets significantly correlated with bacterial density and activity of inflammation, whereas no such correlations were found using the 271 bp PCR primer set. The 139 and 832 bp PCR primer sets concordantly detected the babA2 gene in 93 cases; however, in comparison to the 832 bp PCR primer set, the 139 bp PCR primer set detected additional 50 babA2 cases, whereas only two 832 bp positive cases were missed. The 271 bp PCR primer set missed 32 babA2 cases that were $832 \mathrm{bp}$ and/or $139 \mathrm{bp}$ PCR positive, but tested solely positive in 109 cases. Interestingly, cloning of a subset of $271 \mathrm{bp}$ PCR positive samples revealed amplification of the $b a b A / B$ gene chimera. Hence, in our opinion, the $271 \mathrm{bp}$ PCR protocol is not a reliable diagnostic tool for detecting the babA2 gene in children. Our results reaffirm previous observations that the use of certain $b a b A 2$ PCR primer sets can significantly impact estimation of the prevalence and clinical relevance of the $H$. pylori babA2 gene in children, suggesting babA2 detection methods should be carefully selected.
\end{abstract}

\section{Introduction}

Adherence factors of Helicobacter pylori are considered one of the most important virulence factors, which enable longterm persistence of the bacteria in the human stomach $[1,2]$. $H$. pylori genome encodes a variety of outer membrane proteins (OMPs), including blood group antigen binding adhesion (BabA) proteins. Three bab paralogues have been described thus far: BabA, BabB, and BabC [3]. BabA is one of the most studied $H$. pylori adhesins, capable of binding to Lewis $^{\mathrm{b}}\left(\mathrm{Le}^{\mathrm{b}}\right)$ and related ABO antigens on gastric epithelial cells [1], which in turn results in increased pathogenicity of $H$. pylori and may play a crucial role in the development of H. pylori related gastric pathology such as severe gastritis, peptic ulcers, and gastric adenocarcinoma [4-7]. In contrast, the role of $\mathrm{BabB}$ and $\mathrm{BabC}$ as adhesins has not been demonstrated yet. The BabA protein is encoded by the babA 2 gene, whereas the $b a b A 1$ gene harbors a 10 bp deletion, resulting in protein's inability to interact with $\mathrm{Le}^{\mathrm{b}}$ antigens [1]. Interestingly, it has been shown that the presence of the $b a b A 2$ gene may not uniformly reflect the functional status of the gene $[3,8]$. Based on the level of BabA protein production, strains can be divided into BabA high producers ( $\mathrm{Le}^{\mathrm{b}}$ binding activity, contain babA2 gene), BabA low producers (lacking Le ${ }^{b}$ binding activity but contain $b a b A 2$ gene), and BabA nonproducers (lacking Le ${ }^{\mathrm{b}}$ binding activity, no detectable babA2 gene) [8].

$b a b A$ and $b a b B$ exhibit substantial $\mathrm{N}$ - and C-terminal identity [1,9]. Recombination events between $b a b A$ and $b a b B$ appear to be relatively common and have previously 
been demonstrated for both East Asian and Western strains [10]. Moreover, these intragenomic translocations between the $b a b A$ and $b a b B$ genes are thought to be the main mechanism of BabA expression regulation [3]. Replacement of first $56 \mathrm{bp}$ in the $5^{\prime}$ region of the $b a b B$ with $b a b A$ gene (e.g., $b a b A / b a b B$ chimera) was first identified in clinical isolates [10] and later also reported during experimental $H$. pylori infection in Rhesus macaques [11]. Somewhat less common are $b a b B / A$ chimera. An analysis of the chimeric $b a b B / A$ gene revealed that the first $47 \mathrm{bp}$ were specific for $b a b B$, followed by a $66 \mathrm{bp}$ fragment that was shared between both $b a b A$ and $b a b B$, while the $3^{\prime}$ region showed higher sequence similarity with $b a b A$. Interestingly, this chimeric $\mathrm{BabB} / \mathrm{A}$ protein was shown to have the $\mathrm{Le}^{\mathrm{b}}$ binding capacity [12]. The plasticity of OMPs, not only across different strains but also within a strain colonizing an individual patient, can be regarded as an evolutionary asset, enabling better adaptation of $H$. pylori to the harsh milieu of the human stomach [13].

Various polymerase chain reaction- (PCR-) based methods have been used to detect the babA2 gene [8]. The majority of studies evaluating the BabA status used PCR primers, designed by Gerhard et al. [4], which aim to detect the $10 \mathrm{bp}$ deletion that distinguishes between the $b a b A 1$ and $b a b A 2$ genes. These primers target the highly polymorphic zone of the $b a b A 2$ gene, amplifying an 832 bp long fragment. Another commonly used PCR primer set was designed by Sheu et al., targeting the promoter region and thus amplifying a $271 \mathrm{bp}$ fragment of the babA2 gene [14].

In our previous study, we evaluated the $b a b A 2$ gene prevalence and clinical relevance in Slovenian children using newly developed primers designed by our research group [15]. Interestingly, while additionally testing a subset of samples with the 832 bp PCR primer set, we noticed considerable differences in the prevalence of the $b a b A 2$ gene depending on the type of PCR primer set used [15]. Hence, in order to determine whether the use of a specific PCR primer set influences the babA2 gene prevalence and clinical relevance in a pediatric population, we performed a head-to-head comparison of babA2-specific PCR primer set targeting the $139 \mathrm{bp}$ of the babA2 gene designed by our research group [15] with two widely used $b a b A 2$ PCR primer sets targeting 832 bp [4] and 271 bp segments [14] of the babA2 gene.

\section{Materials and Methods}

2.1. Patients. The study protocol has been described in detail previously [15-17]. Briefly, gastric biopsy samples were obtained from 279 consecutive $H$. pylori-positive children (107 male, 172 female; age range, 5 months-18 years) who had undergone upper endoscopy in the University Children's Hospital in Ljubljana, Slovenia, during the period 1999-2011. Exclusion criteria included previous antibiotic treatment of $H$. pylori infection and usage of nonsteroidal anti-inflammatory drugs, proton pump inhibitors, or $\mathrm{H} 2$ receptor blockers within 30 days prior to the endoscopic examination.
2.2. Histology. At endoscopy, four biopsy samples were obtained from the gastric antrum and corpus from each patient. Two biopsy samples were used for rapid urease testing (H. pylori Quick Test; Biohit Diagnostics, Helsinki, Finland) and the rest were subjected to histopathological examination [16, 17]. According to the updated Sydney classification, two experienced pathologists evaluated the following histological features: density of $H$. pylori colonization, activity of inflammation, chronic inflammation, atrophy, and intestinal metaplasia [18].

2.3. H. pylori DNA Extraction and babA2 Genotyping. As described in detail previously $[16,17], H$. pylori DNA was extracted from the biopsy samples used for the rapid urease testing using the QIAamp DNA Mini kit (Qiagen $\mathrm{GmbH}$, Hilden, Germany). The detection of the babA2 gene was performed in all $H$. pylori samples using a PCR primer set targeting a $139 \mathrm{bp}$ fragment of the central region of $b a b A 2$ that was designed by our research group [15] and two widely used PCRs, targeting $832 \mathrm{bp} \mathrm{[4]} \mathrm{and} 271 \mathrm{bp}$ [14] fragments of a $5^{\prime}$ part of babA2 (Table 1 ).

The 139 bp babA2 PCR protocol was performed using a HotStarTaq Plus DNA Polymerase kit (Qiagen, Hilden, Germany), as described previously [15]. The 832 and $271 \mathrm{bp}$ PCR protocols were performed using AmpliTaq Gold DNA Polymerase kit (Applied Biosystems, Foster City, CA, USA) and FastStart Taq DNA Polymerase kit (Roche, Basel, Switzerland) as described previously [4, 14]. The resulting $139 \mathrm{bp}$ and $832 \mathrm{bp}$ amplicons were analyzed on a $2 \%$ agarose gel and only a subset of amplicons (15 PCR products per each PCR protocol) was sequenced with the same primers as those used for initial PCR [19] because both PCR protocols have already been extensively evaluated in previous studies $[4,15]$.

Sequencing of 13 randomly selected solely 271 bp PCR positive samples and two samples that tested positive using all three PCRs was attempted; however, we were not able to obtain the respective sequences due to technical difficulties. Hence, these samples were purified using QIAquick PCR Purification kit (Qiagen) and subsequently cloned, using CloneJET PCR Cloning Kit (Thermo Fisher Scientific, Vilnius, Lithuania). A sticky-end cloning protocol was used and the overnight transformation was performed using One Shot ${ }^{\circledR}$ TOP10 Chemically Competent E. coli (Invitrogen, Carlsbad, USA), according to the manufacturer's instructions. Recombinant plasmid clones from the transformed bacterial culture were verified for the corresponding H. pylori inserts with colony PCR and sequencing of PCR products performed using pJET1.2 sequencing primers (pJET1.2 F 5'-CGACTCACTATAGGGAGAGCGGC-3' ${ }^{\prime}$ and pJET1.2 R 5'-AAGAACATCGATTTTCCATGGCAG-3'), according to the manufacturer's instructions.

The obtained sequences were annotated by BLAST nucleotide search against the current NCBI database. The sequences were aligned and edited using BioEdit ClustalW multiple sequence alignment together with $b a b A 2$ and $b a b B$ reference sequences obtained from publically available NCBI database. Phylogenetic analysis was performed using PhyML 
TABLE 1: PCR primers used for the babA2 gene detection.

\begin{tabular}{|c|c|c|c|}
\hline Primer & Sequence $\left(5^{\prime}-3^{\prime}\right)$ & Size (bp) & Reference \\
\hline $\begin{array}{l}\text { babA2F } \\
\text { babA2R }\end{array}$ & $\begin{array}{c}\text { AATCCAAAAAGGAGAAAAAGTATGAAA (s) } \\
\text { TGTTAGTGATTTCGGTGTAGGACA (as) }\end{array}$ & 832 & Gerhard et al. [4] \\
\hline $\begin{array}{l}\text { bab7-F } \\
\text { bab7-R }\end{array}$ & $\begin{array}{l}\text { CCAAACGAAACAAAAAGCGT (s) } \\
\text { GCTTGTGTAAAAGCCGTCGT (as) }\end{array}$ & 271 & Sheu et al. [14] \\
\hline $\begin{array}{l}\text { babA (B)-F1 } \\
\text { babA (B)-F2 } \\
\text { babA (B)-F3 } \\
\text { babA2k-rew2 }\end{array}$ & $\begin{array}{l}\text { TATCAAGCCGTGCTTTT (s) } \\
\text { TATCAGGCCGTGCTTTT (s) } \\
\text { TATCAAGCGGTGCTTTT (s) } \\
\text { CAACGAGCCAGGGTATC (as) }\end{array}$ & 139 & Homan et al. [15] \\
\hline
\end{tabular}

s: sense; as: antisense.

(v3.0) integrated in the Phylogeny.fr platform, including HKY85 as a substitution model with 4 gamma categories. The proportion of invariable sites and gamma shape parameter were estimated directly from the data. The obtained phylogenetic tree was graphically presented with Figtree version 1.4 .3 (http://tree.bio.ed.ac.uk/software/figtree/) and phylogenetic relationships were assessed according to the approximate likelihood ratio test (aLRT) branch support values [20].

In addition, because some of the sequences obtained exhibited mismatch with the babA2 reference sequences, amplification of $b a b A / B$ chimera was suspected. The presence of chimeric $b a b A / B$ gene was studied by performing bootscanning method available in Simplot, version 3.5.1., with 500 bootstrap replicates on a varying window sizes (120-60 bp) with a $10 \mathrm{bp}$ sliding window [21]. Two separate slices of alignments were generated and phylogenetic analysis was performed, as described above.

2.4. Statistical Analysis. The data were analyzed using the SPSS 11.0 statistical package (SPSS GmbH Software, Munich, Germany). Statistical significance of differences between evaluated PCR primer sets was evaluated using the $t$-test and Chi square test with a $p$ value of less than 0.05 considered as statistically significant.

\section{Results}

3.1. Prevalence of the babA2 Gene. Using $832 \mathrm{bp}$ [4], $139 \mathrm{bp}$ [15], and 271 bp [14] PCR primer sets, babA2 was detected in $34.0 \%$ (95/279), 51.3\% (143/279), and 79.6\% (222/279) of $H$. pylori samples studied, respectively. Concordant results (either all positive or negative for babA2) were obtained in $35.5 \%(99 / 279)$.

As shown in Figure 1, the 139 bp and 832 bp babA2 PCR primer sets concordantly detected babA2 in 93 cases; however, in comparison to the 832 bp PCR primer set, the 139 bp PCR primer set additionally detected 50 babA2positive samples while missing two samples that were babA2 positive according to the $832 \mathrm{bp}$ PCR primer set. In total, 109 samples were concordantly negative using the 832 bp and 139 bp PCR primer sets but tested positive using the $271 \mathrm{bp}$ PCR primer set. Furthermore, the $271 \mathrm{bp}$ PCR primer set missed $32 H$. pylori samples that tested positive using the 139 bp and/or 832 bp PCR primer sets.

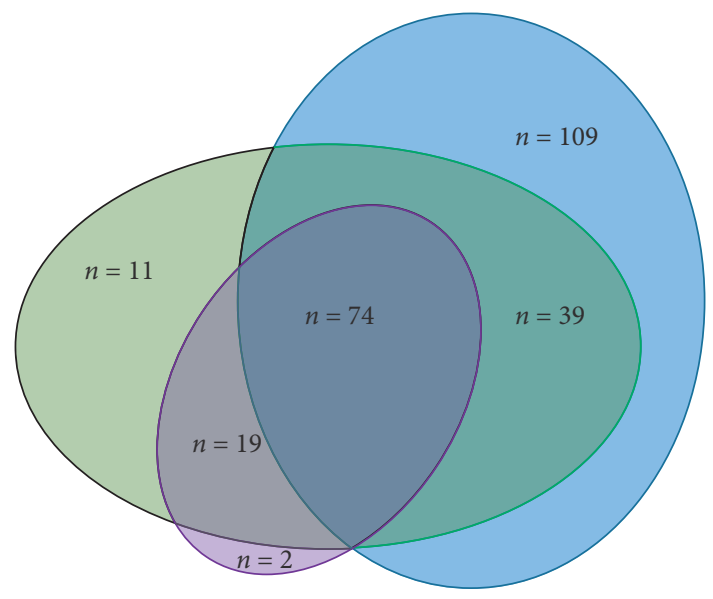

Figure 1: Venn diagram showing all possible combinations of babA2 positive strains determined by the $832 \mathrm{bp}$ (purple), $139 \mathrm{bp}$ (green), and 271 bp (blue) babA2 PCR protocols.

3.2. Association of babA2 Genotypes with Histological Parameters in Gastric Mucosa. In order to evaluate to what extent a certain babA2 PCR protocol affects result interpretation on the clinical relevance of the $b a b A 2$ gene, we assessed potential correlations between the presence of the $b a b A 2$ gene detected by three different PCR protocols and five histological parameters. As shown in Table 2, $H$. pylori strains determined as babA2 positive by the 139 bp PCR protocol were strongly associated with two out of five histological parameters studied: $H$. pylori density score $(p=0.007)$ and activity of inflammation $(p=0.003)$, while the association with chronic inflammation of the gastric mucosa was borderline $(p=0.063)$. Similarly, when using the 832 bp PCR primer set, a strong association was observed between the $H$. pylori strains determined as babA2 positive and $H$. pylori density score $(p=0.015)$ and activity of inflammation $(p=0.016)$ (Table 2 ). In contrast, $b a b A 2$ positive strains confirmed by the 271 bp PCR showed no statistically significant associations with any of the five histological parameters evaluated (Table 2).

3.3. Amplicon Sequencing, Cloning, and Phylogeny. Sanger sequencing of a subset (15 cases each) of randomly selected $139 \mathrm{bp}$ and $832 \mathrm{bp}$ amplicons confirmed the presence of babA2-specific sequences. However, when using the $271 \mathrm{bp}$ 
TABle 2: Association between the presence of the babA2 gene determined by $823 \mathrm{bp}, 139 \mathrm{bp}$, and $271 \mathrm{bp}$ PCR primer sets and histological parameters.

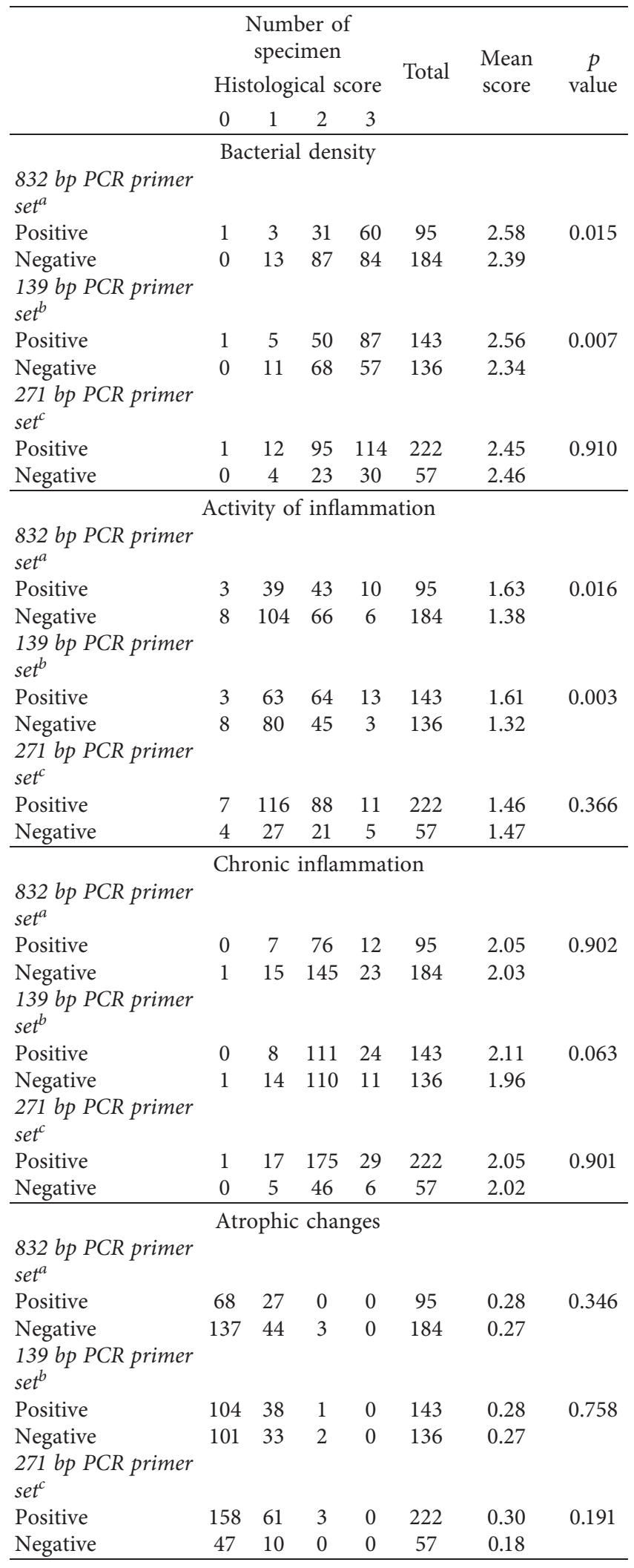

TABle 2: Continued.

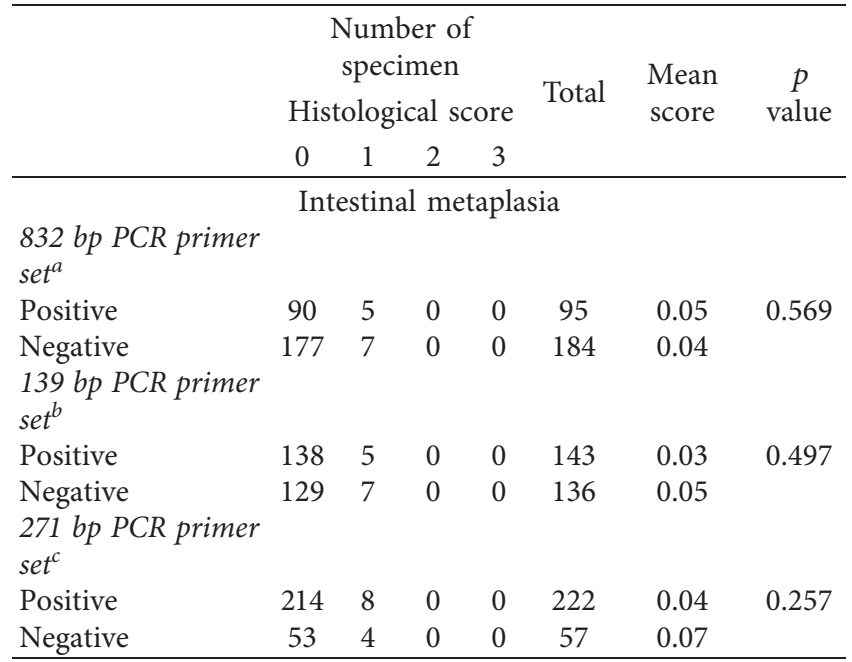

${ }^{a}$ Gerhard et al. [4]; ${ }^{b}$ Homan et al. [15]; ${ }^{c}$ Sheu et al. [14].

PCR primer set, the amplicons showed substantial diversity in amplicon length, ranging from approximately $240 \mathrm{bp}$ to $300 \mathrm{bp}$. Direct sequencing of these variable PCR amplicons was attempted; however, we were unable to obtain complete DNA sequences, most likely due to the presence of repetitive elements. Hence, cloning of 15 randomly selected $271 \mathrm{bp}$ PCR positive samples with amplicon sizes not corresponding to $271 \mathrm{bp}$ was performed. The following cases were evaluated: 13 samples that were solely positive using the $271 \mathrm{bp} \mathrm{PCR} \mathrm{(samples}$ HP-151, HP-194, HP-208, HP-209, HP-214, HP-267, HP271, HP-272, HP-276, HP-283, HP-294, HP-295, and HP296), and two samples that were positive using all three PCR protocols (samples HP-219 and HP-284). The phylogenetic analysis (Figure 2) of the obtained sequences showed clustering into two distinct groups with significant aLRT branch support values of more than 0.99 . Namely, $9 / 15$ sequences clustered together with the babA2 gene reference sequences, while $6 / 15$ sequences clustered with the $b a b B$ gene references.

Simplot analysis indicated possible evidence of recombination (Figure 3), and therefore separate phylogenetic analyses were performed for two slices of the alignment (1-156 bp and 157-246 bp), according to the average recombination breakpoint of analyzed samples. The obtained phylogenetic tree of the first section of the alignment displayed phylogenetic clustering of all 15 generated sequences with the $b a b A 2$ reference sequences, although with less significant aLRT value (0.787) (Figure 4). On the other hand, the second part of the alignment displayed the same phylogenetic relationship, as did the previous phylogenetic analysis performed on the complete alignment (Figure 2). Therefore, our results indicate that the $271 \mathrm{bp}$ primers set amplified chimeric $b a b A / B$ gene. 


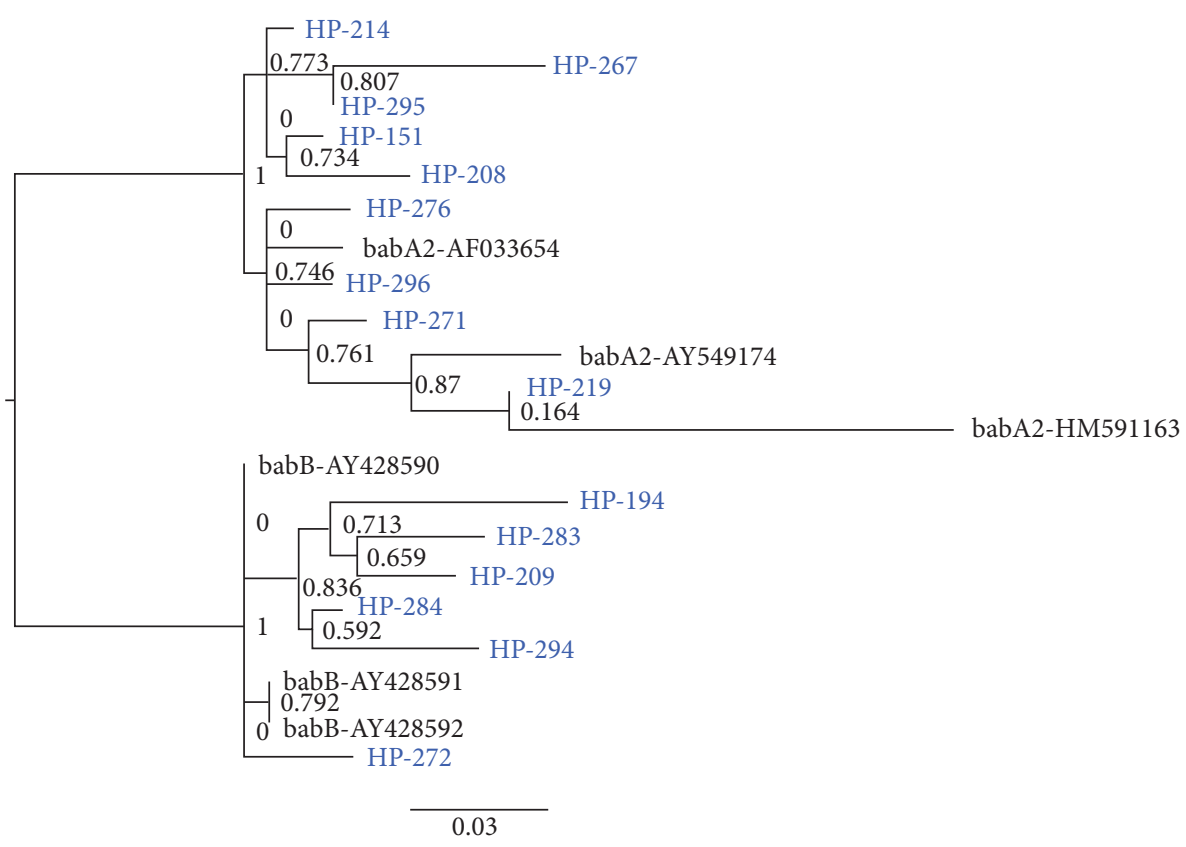

FIGURE 2: Phylogenetic tree showing three $b a b A 2$ and three $b a b B$ gene reference sequences and 15 strains, which tested positive with the 271 bp PCR primer set [14]. Numbers at nodes show aLRT branch support values. H. pylori isolates are named as HP-consecutive number (blue), the reference $b a b A 2$ and $b a b B$ gene sequences, obtained from publically available NCBI database, are provided with the respective accession numbers (black).

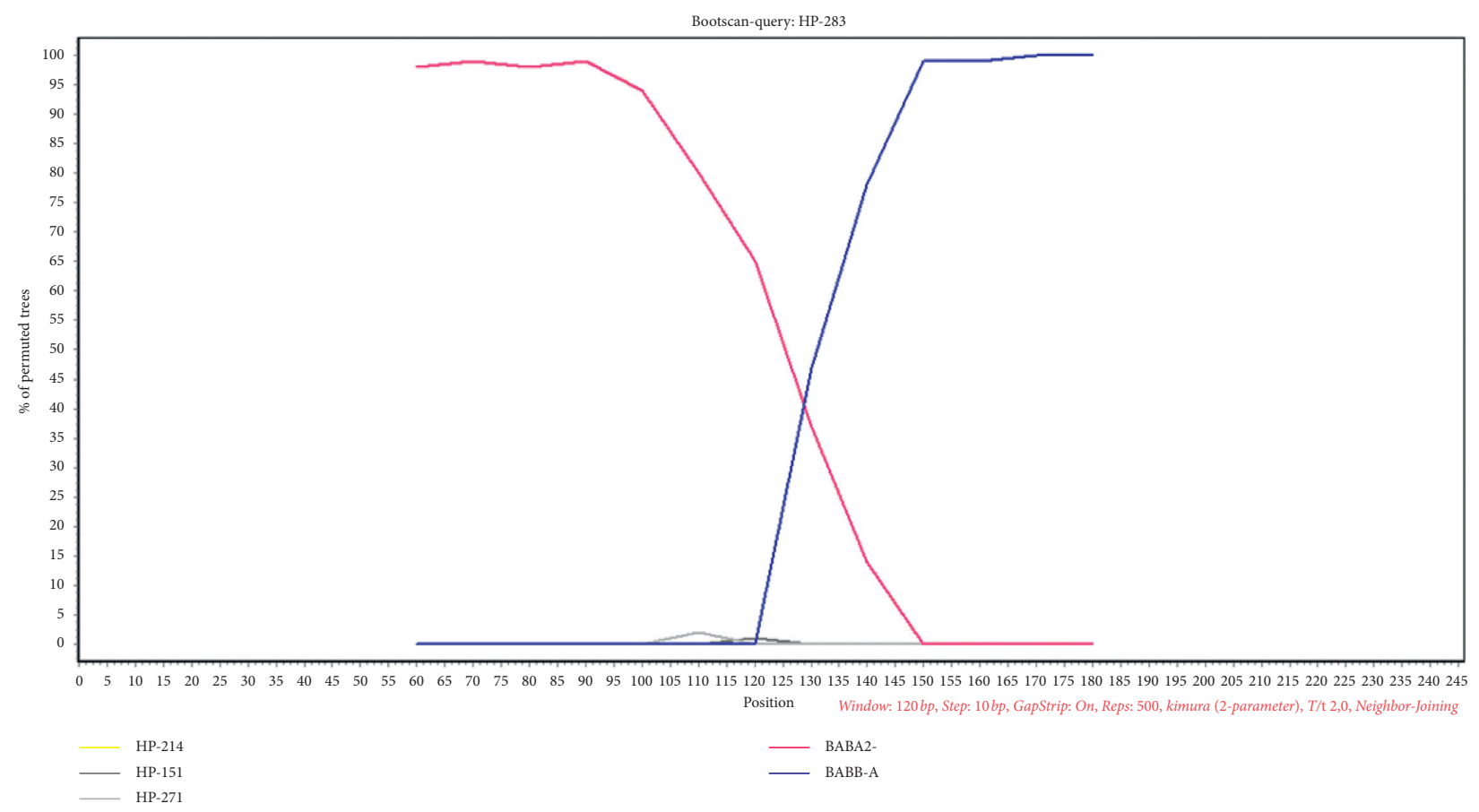

Figure 3: Analysis of the $b a b A$ and $b a b B$ gene recombination. Bootscan of sample HP-283 is presented using Simplot with 500 bootstrap replicates, window size of $120 \mathrm{bp}$ and $10 \mathrm{bp}$ sliding window.

\section{Discussion}

In this study, we performed a head-to-head comparison of babA2 gene-specific PCR primer set designed by our research group [15] with two previously established babA2 PCR protocols in order to assess the $b a b A 2$ prevalence using the largest collection of $H$. pylori positive biopsies obtained from children $(n=279)$. Interestingly, the prevalence of the babA2 gene in our study varied significantly according to the PCR primer set used; the $b a b A 2$ positivity rate using the 271 bp PCR primer set was more than double of that obtained by the 832 bp PCR primer set (79.6\% versus $34.0 \%$ ), whereas the 


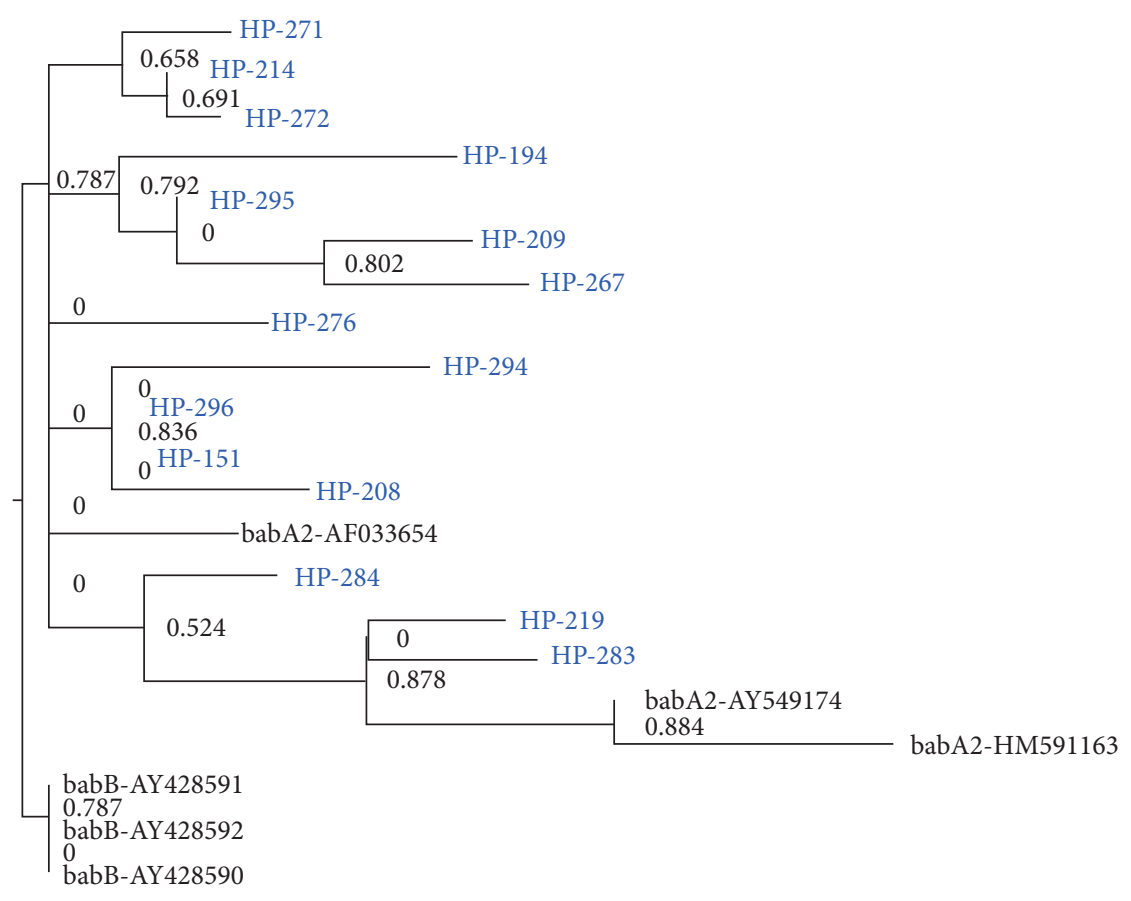

0.02

Figure 4: Phylogenetic tree of the first section (1-156 bp) of the alignment showing phylogenetic clustering of 15 generated sequences with the $b a b A 2$ reference sequences. $H$. pylori isolates are named as HP-consecutive number (blue), the reference $b a b A 2$ and $b a b B$ gene sequences, obtained from publically available NCBI database, are provided with the respective accession numbers (black).

139 bp PCR primer set yielded intermediate babA2 positivity rates $(51.3 \%)$. In majority of previous studies in adults and children [4, 6, 22-30], the babA2 status was determined using the 832 bp PCR primer set, originally designed by Gerhard et al. [4]. According to the literature data, the prevalence of the $b a b A 2$ gene in adults determined by using these primers is highly variable, ranging from 32 to $72 \%$ in Western countries and from 64 to $85 \%$ in Asian countries [8], while in children, the babA2 gene positivity ranges from 17 to $84 \%$ [22-30]. Sheu et al. [14] designed a second widely used PCR primer set, which amplifies a $271 \mathrm{bp}$ fragment of the $b a b A 2$ gene. In comparison to the 832 bp PCR primer set, the $b a b A 2$ prevalence determined using the 271 bp PCR primer set in adults was generally higher, ranging from 41 to $100 \%[7,14,31,32]$. To the best of our knowledge, only one previous study used the $271 \mathrm{bp}$ PCR primer set for detecting the babA2 gene in children, yielding a $41 \%$ prevalence among Turkish pediatric population [33].

Adherence of $H$. pylori to gastric epithelial cells is one of the most important contributing factors to the pathogenicity of the bacteria [34]. The $b a b A 2$ gene encodes a protein that enables $H$. pylori attachment to $\mathrm{Le}^{\mathrm{b}}$ epitopes on human epithelial cells and thus the delivery of bacterial virulence factors into host target cells, resulting in gastric tissue damage [35]. In this study, we also aimed to evaluate whether the use of certain $b a b A 2$ PCR primer set influences the clinical relevance of the $b a b A 2$ gene. Interestingly, we found a strong correlation between $b a b A 2$ positive status and the density of $H$. pylori colonization, as well as the degree of active inflammation determined by the 139 and 832 bp PCR primer sets (Table 2). However, no significant associations were found between the babA2 status and H. pylori density score, degree of activity, or chronic inflammation of the gastric mucosa when using the $271 \mathrm{bp}$ PCR primer sets. In this study, babA2 positive strains (determined with any of the three PCR methods used) were not found more frequently in patients with gastric atrophy or intestinal metaplasia, suggesting involvement of other virulence or host factors in the disease progression. Nevertheless, data regarding the babA2 gene prevalence and clinical significance should be interpreted with caution, since the use of a particular babA2 PCR primer set results in significant differences in the $b a b A 2$ gene detection rates. Moreover, conflicting results on the association between the presence of the babA2 gene and gastric cancer obtained in previous studies could be due to using only a single PCR primer set for detection of the $b a b A 2$ gene, an approach that may not reflect the actual status of the BabA protein expression [35]. Chang et al. [36] even proposed using multiple pairs of PCR primers to provide a more reliable estimation of the BabA status and its influence on the risk of gastric cancer, especially in isolates from East Asia with nearly 100\% prevalence of the babA2 gene $[14,36]$.

The $b a b A$ gene was initially cloned from $H$. pylori strain CCUG17875, which contains a silent (nonfunctional) $b a b A 1$ gene and an expressed $b a b A 2$ gene. The sequences of these two genes differ only by the presence of a $10 \mathrm{bp}$ deletion in the signal peptide sequence of $b a b A 1$, which eliminates its 
translational initiation codon. However, later analysis showed that naturally occurring $b a b A 1$ sequences are very rare, suggesting PCR-based methods designed to detect the 10 bp deletion (e.g., the 832 bp PCR primer set) do not reliably reflect the BabA status $[8,13]$. As shown in Figure 1, the 139 bp PCR primer set detected 50 additional babA2 positive cases compared to the widely used 832 bp PCR primer set, suggesting superior sensitivity of the $139 \mathrm{bp}$ PCR primer set for detecting the $b a b A 2$ gene. Thus, our data support previous observations of ineffectiveness and underestimation of the $b a b A 2$ gene prevalence when using the 832 bp PCR primer set $[6,8,22]$. Although the forward babA2F primer is located within a relatively conserved $5^{\prime}$ region, the presence of single nucleotide polymorphisms may nonetheless impede effective amplification. Moreover, because the reverse babA2R primer was designed to anneal within a highly variable region in order to reliably discriminate between various $b a b$ homologues, this may invariably lead to underestimation of the babA2 prevalence [6]. According to Fujimoto et al. [8], the sensitivity of the $b a b A 2$ PCR primer sets that were designed to discriminate between the $b a b A 2$ and $b a b A 1$ genes was shown to be only approximately $36-49 \%$, thus missing a significant proportion of the BabA high and low producers. Nevertheless, significantly higher prevalence of the babA2 gene (e.g., $>70 \%$ ) obtained from Asian isolates using the same primer set highlights the significance of geographical variability among $H$. pylori strains $[6,8,22]$.

The issue of nucleotide mismatching in the primer binding region of the 832 bp PCR primer set was circumvented when designing primers for the $139 \mathrm{bp}$ PCR protocol by systematic investigation of the $b a b A 2$ genomic diversity of geographically distinct $H$. pylori samples and the consequent addition of more $b a b A 2$ specific primers to cover all variations of the $b a b A 2$ gene known at the time of primer design. In specific, three forward and one reverse primer were designed on the basis of multiple alignments of 94 babA2 and 24 babB sequences [15]. To avoid amplifying nearly identical $5^{\prime}$ and $3^{\prime}$ regions of the $b a b A$ and its paralogue $b a b B$, a set of three forward and one reverse primer was selected in the central region of the $b a b A 2$ gene which target conserved regions of the $b a b A 2$, yielding a 139 bp babA2 gene-specific amplicon. However, despite our best efforts to design a PCR primer set that would amplify all babA2 gene variants, the 139 bp PCR primer set failed to detect two $b a b A 2$ positive cases determined by the 832 bp PCR primer set, suggesting addition of more primers or use of a combined approach (e.g., concurrent amplification with the 139 and 832 bp PCR primer sets) may be used in order to increase the sensitivity of the PCR. Nevertheless, as shown by Pride et al. [37], sequencing of the $b a b A$ and $b a b B$ genes from $H$. pylori strains from around the world showed high sequence variability in different strains, thus making it difficult to construct a universally applicable primer set.

The $271 \mathrm{bp}$ PCR primer set uses a forward bab7-F primer that is located in the promoter region of the $b a b A 2$ gene in combination with a unique reverse primer from the $5^{\prime}$ region of the babA2 gene. A study by Fujimoto et al. [8] showed that the 271 bp PCR primer set originally developed by Sheu et al.
[14] is not a reliable molecular tool for detecting the $b a b A 2$ gene in adults, because it frequently amplifies also the $b a b B$ gene sequences and, consequently, produces $b a b A 2$ falsepositive results. Interestingly, the reverse bab7- $\mathrm{R}$ primer sequence matches exactly both the $b a b A 2$ and $b a b B$ gene sequences, which may be the reason for the occasional nonspecific $b a b B$ gene amplification. Our study showed that the inadequacy of the $271 \mathrm{bp}$ PCR for detecting the $b a b A 2$ gene also exists in pediatric population. In this study, Sheu's primers generated not only the predicted 271 bp amplicons, but also amplicons of various sizes, ranging from approximately 240 to $300 \mathrm{bp}$, which was already noted previously [32]. Unlike the study by Mattar et al. [32], in which the two randomly sequenced PCR amplicons were babA2 in origin, $6 / 15$ cloned sequences from our study were shown to belong to the $b a b B$ cluster, whereas the rest of the sequences showed higher similarity to the $b a b A 2$ gene sequences. As shown in Figure 2, significant aLRT branch support values were observed, thus clearly demonstrating clustering of the strains into two distinct groups: the $b a b A 2$ and $b a b B$ gene group. To our surprise, sequencing of one out of two cloned $271 \mathrm{bp}$ amplicons that tested positive using all three PCR protocols yielded $b a b B$-like sequences. Indeed, further analysis revealed that the $271 \mathrm{bp}$ PCR primer set in fact detected $b a b A / B$ gene chimeras (Figure 3 ), which appear to be relatively common among bacterial isolates [38]. It has previously been shown that, in isolates lacking the $b a b A$ gene $(11 / 34 ; 33 \%)$, the chimeric $b a b A / B$ gene which results in loss of $\mathrm{Le}^{\mathrm{b}}$ activity due to inhibition of the BabA expression predominates chimeric $b a b B / A$ gene that otherwise subjects protein expression to phase variation [38]. Furthermore, 32 H. pylori samples that were positive with the $139 \mathrm{bp}$ and/or 832 bp PCR primer sets tested negative with the 271 bp PCR primer set, indicating that this PCR protocol may also generate $b a b A 2$ false-negative results (Figure 1). This may be due to significant variations in $b a b A 2$ gene sequences among the isolates.

To the best of our knowledge, no PCR-based method can be considered as "gold standard" for detecting the babA2 gene. Moreover, PCR-based determination of the functional status of the gene is importantly hampered by several factors, namely varying sensitivity and specificity of different $b a b A 2$ PCR primer sets, also reflecting a high degree of heterogeneity among different $H$. pylori isolates, as well as the presence of BabA low producers: $H$. pylori strains that in spite of detectable $b a b A 2$ gene lack $\mathrm{Le}^{\mathrm{b}}$ binding activity $[3,8]$. Despite being relatively inexpensive and, most importantly, easy to perform and to interpret, PCR-based methods will probably be soon replaced by whole-genome sequencing. It has already been shown that whole-genome sequencing can be used for precise and fast characterization of $H$. pylori virulence genes, including babA2 [39].

There are some limitations to this study, including the relatively low number of sequenced $271 \mathrm{bp}$ PCR amplicons. In addition, determining which $b a b$ gene occupies which genomic locus would also provide additional information regarding the usefulness of respective babA2 PCR primer sets. Because PCRs were performed using tissue biopsies and not $H$. pylori isolates, it is likely that $H$. pylori subpopulations 
were not adequately evaluated. Hence, future studies evaluating these PCR primer sets should be performed on multiple $H$. pylori isolates obtained from various gastric biopsies.

\section{Conclusions}

This study showed that the estimation of the prevalence and clinical relevance of the $H$. pylori babA2 gene in children mainly depends on the PCR primer set used. The 139 bp PCR primer set exhibited superior sensitivity than the previously most frequently used PCR primer set targeting $832 \mathrm{bp}$ fragment of the babA2 gene. Similar to the 832 bp PCR primer set, the babA2 status determined by the 139 bp PCR primer set statistically significantly correlated with two out of five histological parameters evaluated, whereas such correlation was not evident when the $271 \mathrm{bp}$ PCR primer set was used. Comparison of three different babA2 PCR primer sets on the largest number of pediatric $H$. pylori samples to date confirmed previous observations that the $271 \mathrm{bp}$ PCR protocol is a not reliable diagnostic tool for the detection of the $b a b A 2$ gene and should thus be avoided.

\section{Abbreviations}

PCR: Polymerase chain reaction

BabA: Blood group antigen binding adhesion

Le $^{\text {b }}$ Lewis ${ }^{b}$

aLRT: Approximate likelihood ratio test

bp: $\quad$ Base pairs.

\section{Data Availability}

The data used to support the findings of this study are included within the article.

\section{Ethical Approval}

The study was approved by the National Medical Ethics Committee at the Slovenian Ministry of Health.

\section{Consent}

Informed consent was obtained from the parents of all the children.

\section{Conflicts of Interest}

The authors declare no conflicts of interest.

\section{Acknowledgments}

The authors would like to thank Boštjan J. Kocjan for his invaluable help with the design of the $139 \mathrm{bp}$ PCR primer set and cloning of the samples.

\section{References}

[1] D. Ilver, A. Arnqvist, J. Ogren et al., "Helicobacter pylori adhesin binding fucosylated histo-blood group antigens revealed by retagging," Science, vol. 279, no. 5349, pp. 373377, 1998.

[2] A. Magalhães and C. A. Reis, "Helicobacter pylori adhesion to gastric epithelial cells is mediated by glycan receptors," Brazilian Journal of Medical and Biological Research, vol. 43, no. 7, pp. 611-618, 2010.

[3] Y. Yamaoka, "Roles of Helicobacter pylori BabA in gastroduodenal pathogenesis," World Journal of Gastroenterology, vol. 14, no. 27, pp. 4265-4272, 2008.

[4] M. Gerhard, N. Lehn, N. Neumayer et al., "Clinical relevance of the Helicobacter pylori gene for blood-group antigenbinding adhesin," Proceedings of the National Academy of Sciences, vol. 96, no. 22, pp. 12778-12783, 1999.

[5] C.-F. Zambon, F. Navaglia, D. Basso, M. Rugge, and M. Plebani, "Helicobacter pylori babA2, cagA, and s1 vacA genes work synergistically in causing intestinal metaplasia," Journal of Clinical Pathology, vol. 56, no. 4, pp. 287-291, 2003.

[6] F. O. Olfat, Q. Zheng, M. Oleastro et al., "Correlation of the Helicobacter pylori adherence factor BabA with duodenal ulcer disease in four European countries," FEMS Immunology \& Medical Microbiology, vol. 44, no. 2, pp. 151-156, 2005.

[7] A. Talebi Bezmin Abadi, T. Taghvaei, A. Mohabbati Mobarez, G. Vaira, and D. Vaira, "High correlation of $b a b A_{2}$-positive strains of Helicobacter pylori with the presence of gastric cancer," Internal and Emergency Medicine, vol. 8, no. 6, pp. 497-501, 2013.

[8] S. Fujimoto, O. Olaniyi Ojo, A. Arnqvist et al., "Helicobacter pylori BabA expression, gastric mucosal injury, and clinical outcome," Clinical Gastroenterology and Hepatology, vol. 5, no. 1, pp. 49-58, 2007.

[9] R. A. Alm, J. Bina, B. M. Andrews, P. Doig, R. E. W. Hancock, and T. J. Trust, "Comparative genomics of Helicobacter pylori: analysis of the outer membrane protein families," Infection and Immunity, vol. 68, no. 7, pp. 4155-4168, 2000.

[10] D. T. Pride and M. J. Blaser, "Concerted evolution between duplicated genetic elements in Helicobacter pylori," Journal of Molecular Biology, vol. 316, no. 3, pp. 629-642, 2002.

[11] J. V. Solnick, L. M. Hansen, N. R. Salama, J. K. Boonjakuakul, and M. Syvanen, "Modification of Helicobacter pylori outer membrane protein expression during experimental infection of rhesus macaques," Proceedings of the National Academy of Sciences, vol. 101, no. 7, pp. 2106-2111, 2004.

[12] A. Bäckström, C. Lundberg, D. Kersulyte, D. E. Berg, T. Borén, and A. Arnqvist, "From the cover: metastability of Helicobacter pylori bab adhesin genes and dynamics in Lewis b antigen binding," Proceedings of the National Academy of Sciences, vol. 101, no. 48, pp. 16923-16928, 2004.

[13] J. C. Colbeck, L. M. Hansen, J. M. Fong, and J. V. Solnick, "Genotypic profile of the outer membrane proteins BabA and BabB in clinical isolates of Helicobacter pylori," Infection and Immunity, vol. 74, no. 7, pp. 4375-4378, 2006.

[14] B.-S. Sheu, S. M. Sheu, H. B. Yang, A. H. Huang, and J. J. Wu, "Host gastric Lewis expression determines the bacterial density of Helicobacter pylori in babA2 genopositive infection," Gut, vol. 52, no. 7, pp. 927-932, 2003.

[15] M. Homan, A. Šterbenc, B. J. Kocjan et al., "Prevalence of the Helicobacter pylori babA2 gene and correlation with the degree of gastritis in infected Slovenian children," Antonie Van Leeuwenhoek, vol. 106, no. 4, pp. 637-645, 2014.

[16] M. Homan, B. Luzar, B. J. Kocjan et al., "Prevalence and clinical relevance of $\operatorname{cagA}, \operatorname{vacA}$, and iceA genotypes of Helicobacter pylori isolated from Slovenian children," Journal of Pediatric Gastroenterology and Nutrition, vol. 49, no. 3, pp. 289-296, 2009. 
[17] A. Šterbenc, M. Poljak, N. Zidar, B. Luzar, and M. Homan, "Prevalence of the Helicobacter pylori hom A and homB genes and their correlation with histological parameters in children," Microb Pathog, vol. 125, pp. 26-32, 2018.

[18] M. F. Dixon, R. M. Genta, J. H. Yardley, and P. Correa, "Classification and grading of gastritis 1994," The American Journal of Surgical Pathology, vol. 20, no. 10, pp. 1161-1181, 1996.

[19] A. R. Platt, R. W. Woodhall, and A. L. George Jr., "Improved DNA sequencing quality and efficiency using an optimized fast cycle sequencing protocol," BioTechniques, vol. 43, no. 1, pp. 58-62, 2007.

[20] A. Dereeper, V. Guignon, G. Blanc et al., "Phylogeny.fr: robust phylogenetic analysis for the non-specialist," Nucleic Acids Research, vol. 36, no. suppl_2, pp. W465-W469, 2008.

[21] K. S. Lole, R. C. Bollinger, R. S. Paranjape et al., "Full-length human immunodeficiency virus type 1 genomes from subtype $\mathrm{C}$-infected seroconverters in India, with evidence of intersubtype recombination," Journal of Virology, vol. 73, no. 1, pp. 152-160, 1999.

[22] L. E. Torres, K. Melián, A. Moreno et al., "Prevalence of vacA, cagA and babA2 genes in Cuban Helicobacter pylori isolates," World Journal of Gastroenterology, vol. 15, no. 2, pp. 204-210, 2009.

[23] M. Oleastro, M. Gerhard, A. I. Lopes et al., "Helicobacter pylori virulence genotypes in Portuguese children and adults with gastroduodenal pathology," European Journal of Clinical Microbiology and Infectious Diseases, vol. 22, no. 2, pp. 85-91, 2003.

[24] R. P. Podzorski, D. S. Podzorski, A. Wuerth, and V. Tolia, "Analysis of the vacA, cagA, cagE, iceA, and babA2 genes in Helicobacter pylori from sixty-one pediatric patients from the Midwestern United States," Diagnostic Microbiology and Infectious Disease, vol. 46, no. 2, pp. 83-88, 2003.

[25] Y. Erzin, V. Koksal, S. Altun et al., "Prevalence of Helicobacter pylori vacA, cagA, cagE, iceA, babA2 genotypes and correlation with clinical outcome in Turkish patients with dyspepsia," Helicobacter, vol. 11, no. 6, pp. 574-580, 2006.

[26] L. Boyanova, D. Yordanov, G. Gergova, R. Markovska, and I. Mitov, "Association of iceA and babA genotypes in Helicobacter pylori strains with patient and strain characteristics," Antonie Van Leeuwenhoek, vol. 98, no. 3, pp. 343-350, 2010.

[27] G. T. Garcia, K. R. S. Aranda, M. E. P. Goncalves et al., "High prevalence of clarithromycin resistance and cagA, vacA, iceA2, and babA2 genotypes of Helicobacter pylori in Brazilian children," Journal of Clinical Microbiology, vol. 48, no. 11, pp. 4266-4268, 2010.

[28] M. Oleastro, A. Santos, R. Cordeiro, B. Nunes, F. Mégraud, and A. Menard, "Clinical relevance and diversity of two homologous genes encoding glycosyltransferases in Helicobacter pylori," Journal of Clinical Microbiology, vol. 48, no. 8 , pp. 2885-2891, 2010

[29] S. Talarico, B. D. Gold, J. Fero et al., "Pediatric Helicobacter pylori isolates display distinct gene coding capacities and virulence gene marker profiles," Journal of Clinical Microbiology, vol. 47, no. 6, pp. 1680-1688, 2009.

[30] S. Mendoza-Elizalde, A. C. Cortés-Márquez, S. Giono-Cerezo et al., "Analysis of the genotypic diversity of strains of $\mathrm{Hel}$ icobacter pylori isolated from pediatric patients in Mexico," Infection, Genetics and Evolution, vol. 29, pp. 68-74, 2015.

[31] H. Shahi, S. Reiisi, R. Bahreini, N. Bagheri, L. Salimzadeh, and H. Shirzad, "Association between Helicobacter pylori cagA, babA2 virulence factors and gastric mucosal interleukin-33 mRNA expression and clinical outcomes in dyspeptic patients," International Journal of Molecular and Cellular Medicine, vol. 4, no. 4, pp. 227-234, 2015.

[32] R. Mattar, A. F. d. Santos, J. N. Eisig et al., "No correlation of bab A2 with vac A and cag A genotypes of Helicobacter pylori and grading of gastritis from peptic ulcer disease patients in Brazil," Helicobacter, vol. 10, no. 6, pp. 601-608, 2005.

[33] G. Ozbey, Y. Dogan, and K. Demiroren, "Prevalence of Helicobacter pylori virulence genotypes among children in Eastern Turkey," World Journal of Gastroenterology, vol. 19, no. 39, pp. 6585-6589, 2013.

[34] D. J. Evans Jr. and D. G. Evans, "Helicobacter pylori adhesins: review and perspectives," Helicobacter, vol. 5, no. 4, pp. 183-195, 2000.

[35] S. Backert, M. Clyne, and N. Tegtmeyer, "Molecular mechanisms of gastric epithelial cell adhesion and injection of CagA by Helicobacter pylori," Cell Communication and Signaling, vol. 9, no. 1, p. 28, 2011.

[36] W.-L. Chang, Y.-C. Yeh, and B.-S. Sheu, "The impacts of $H$. pylori virulence factors on the development of gastroduodenal diseases," Journal of Biomedical Science, vol. 25, no. 1, p. 68, 2018.

[37] D. T. Pride, R. J. Meinersmann, and M. J. Blaser, "Allelic variation within Helicobacter pylori babA and babB," Infection and Immunity, vol. 69, no. 2, pp. 1160-1171, 2001.

[38] M. J. Matteo, R. I. Armitano, M. Romeo, A. Wonaga, M. Olmos, and M. Catalano, "Helicobacter pylori bab genes during chronic colonization," International Journal of Molecular Epidemiology and Genetics, vol. 2, no. 3, pp. 286-291, 2011.

[39] F. Imkamp, F. N. Lauener, D. Pohl et al., "Rapid characterization of virulence determinants in Helicobacter pylori isolated from non-atrophic gastritis patients by next-generation sequencing," Journal of Clinical Medicine, vol. 8, no. 7, p. 1030, 2019. 